suivante, c'est-à-dire que le plasma du lait eontient une agglutinine qui agit sur les membranes des globules de matière grasse, et que cette enzyme est tout bonnement détruite par le chauffage du lait. Précisément, les modes de chauffage qui détruisent la montée de la crème du lait détruisent aussi l'agglutinine du sang de bœuf, et comme des investigateurs hollandais ont déjà montré que le sérum du sang de bœuf, et spécialement l'euglobuline qui y est contenue (1) favorise la faculté de montée de la crème du lait, il ne peut y avoir absolument aucun doute à ce que l'agglutinine, ou les agglutinines du lait proviennent du sérum du sang. Comme les agglutinines qui agglutinent les globules rouges du sang ont une température optimum basse, nous avons d'une manière toutà fait spontanós l'explication du phénomène remarquable qui est que la montée de la crème a lieu plus facilement à basse température, quoique la viscosité du plasma du lait soit d'autant plus grande, que la température est plus basse.

Il ne faut pas oublier que, dans cette étude, quand on parle de montée de erème, on ne tient compte que de celle qui a lieu pendant 2 heures dans les conditions indiquées. En 24 heures et même en plus de temps, une partie importante des globules de matière grasse non agglutinés atteignent la couche de crème, de sorte que les différences dans la faculté de montée de la crème, sur lesquelles nous avons basé notre réaction, disparaissent partiellement. Avec le lait de Jersey, cette altération du rapport A pout déjà a voir lieu avant que les deux heures ne se soient écoulées, car les globules de matière grasse de ce lait sont tellement gros qu'ils atteignent rapidement la surface sans être agglomérés ensemble. C'est ce qui explique pourquoi notre réaction de montée de crème ne convient pas si bien pour le lait de Jersey. Par la centrifugation, les globules de matière grasse sont soumis à de si grandes forces que cela ne signifie rien, qu'ils soient agglutinés ou non, et de nombreuses masses de globules gras seront tout de même réduites en morceaux par le traitement mécanique violent, auquel elles sont soumises. Ici, il n'y a que la viscosité du plasma du lait qui semble jouer un rôle, et e'est par la centrifugation qu'on obtient tout de même le meilleur écrémage à des températures qui sont extrêmement nuisibles à la montée naturelle de la crème du lait.

(A suivre.)

\title{
AVANTAGES DES POMPES A ENGRENAGE POUR LA CIRCULATION DU LAIT, DE LA BIËE DES JUS DE FRUITS \\ DANS LES APPAREILS DE PASTEURISATION
}

\author{
par H. STASSANO et A. P. ROLLET.
}

L'un de nous a été amené à adopter le système de la pompe à engre-

(1) VAN DAM, HEKMA, SiRzs et BrouWER (Verslagen Landbouwlound. Onderz. d. Rijks. landbouwproefstation, 1922, 1923, 1924 et 1925). 
nage pour faire circuler le lait dans les appareils de pasteurisation de lait en couche mince à la place de tout autre système de pompe, partieulièrement du système à piston, jugeant $a$ priori que dans le premier eas seulement le liquide est sans cesse entraîné par les dents de l'engrenage qui se succèdent à de très courts intervalles, pour le happer au passage et le déverser, par l'ouverture opposée, en un flux continu. Dans les autres systèmes de pompes, des raréfactions d'air doivent également se produire, mais beaucoup plus fortes, et avec de bien moindre fréquence, d'où la plus grande possibilité de troubles dans l'équilibre gazeux du liquide sur lequel on opère.

L'expérience montra qu'effectivement par l'emploi d'une petite pompe à engrenage actionnant la circulation du lait dans un pasteurisateur d'essai de 350 litres de débit par heure, on ne perdait pas la moindre trace de l'acide carbonique renfermé dans le lait cru, ce qui constitue l'un des principaux avantages du traitement thermique du lait sous couche mince, en circuit fermé, sur les procédés usuels où cette perte par contre est très considérable. Par le procédé de pasteurisation basse, il ne reste en moyenne dans le lait que $14,1 \mathrm{~cm}^{3}$ sur la moyenne du lait cru qui est de $43,29 \mathrm{~cm}^{3}$ par litre, et par le procédé de pasteurisation haute à psine $4,3 \mathrm{~cm}^{3}$. De plus, comme nous l'avons déjà signalé (I): "L'analyse des gaz extraits par l'action du vide sur les différents laits montre que, parallèlement à cette perte en acide carbonique, le lait s'enrichit"(dans les procédés usuels ci-dessus, ainsi que dans le chauffage en général à l'air libre) en oxygène et en azote d'autant plus qu'il a été chauffé davantage. Par contre, dans les laits pasteurisés sous couche mince et en circuit fermé, où nous avons déjà montré qu'il ne se produisait aucun départ d'acide carbonique, l'analyse des gaz montre qu'il n'y a pas non plus aération et même plutôt dans quelques cas, il se produit une légère perte en oxygène et azote. »

Or, lorsque l'on passa du petit appareil d'essai, où la circulation du lait était réalisée au moyen d'une petite pompe à engrenage, à l'appareil industriel en exercice à la Laiterie Centrale de Strasbourg de 2.000 litres de débit, dans lequel cette circulation a été effectuée pendant deux ans à l'aide d'une pompe à 4 pistons actionnés alternativement par un excentrique (pompe que le constructeur de Paris fournit en prétendant qu'elle conviendrait même davantage surtout pour soutenir l'effort de la pression); nous constatâmes que l'on perdait presque la moitié de l'acide carbonique contenu normalement dans le lait. Nous ne retrouvâmes effectivement que $21,6 \mathrm{~cm}^{3}$ sur la moyenne du lait cru qui est de $43,29 \mathrm{~cm}^{3}$ d'après nos propres analyses.

Il fallut se servir néanmoins de cette pompe jusqu'à ce qu'elle se fut usée, ce qui s'est produit même assez prématurément, au bout de

${ }^{1}$ C. R. Acadómie des Seienees 1924, t, CLXXIX, p. 438, et C. R. Socí́té de Biologie" t. XCIII, p. 716. 
deux ans seulement. La nouvelle pompe qui vient d'être installée à sa place, et à titre d'essai, est naturellement du type à engrenage, et, pøur préciser, à dents inclinées hélicoïdales.

Voici les chiffres obtenus dans l'analyse du lait porté à $74^{\circ}$ et qui circula au moyen de la pompe en question. En regard des chiffres correspondant au lait traité par le procédé de la couche mince (stassanisé) dans ces conditions, nous marquons les chiffres qui correspondent au lait témoin cru.

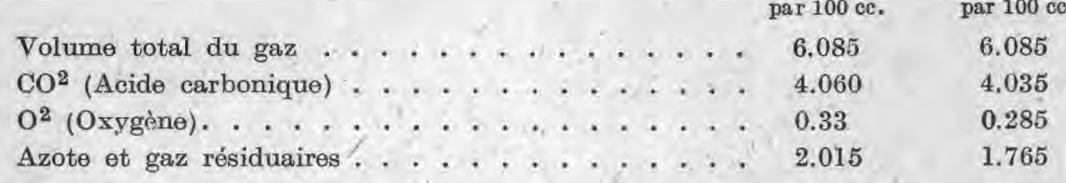

Un simple regard donné à ces deux colonnes montre non seulement que par la substitution de la nouvelle pompe à engrenage à l'ancienne à piston, au même grand appareil de pasteurisation de la laiterie, sans qu'aucun autre changement y ait été apporté, la perte de l'acide carbo: nique a été réduite à une valeur négligeable; mais que de plus on a obtenu encore l'autre avantage de même nature mentionné plus haut du procédé de la couche mince, à savoir l'absence d'aération accompagnée encore d'une légère perte en oxygène et en azote, e'est-à-dire de l'air dissous dans le lait depuis la sortie du pis de la vache jusqu'au moment de la pasteurisation.

Ces résultats sont tels qu'ils nous dispensent d'insister davantage sur la nécessité de l'emploi de la pompe à engrenage pour conserver au procédé de la pasteurisation du lait en circulation continue, sous couche mince, l'un de ses plus utiles avantages sur les procédés usuels, à savoir: la conservation de l'acide carbonique conjointement à l'absence d'aération.

Le besoin d'éviter l'aération se fait sentir également quoique à un autre égard dans la pasteurisation du vin, du cidre, des moûts et en général des jus de fruits.

Pour le lait, l'inconvénient de l'aération pendant la pasteurisation porte sur les vitamines, lesquelles sont particulièrement sensibles à l'action du chauffage en présence de l'oxygène de l'air.

Pour les vins, les cidres, les moûts et les jus de fruits en général, l'inconvénient de l'aération de ces liquides pendant le chauffage affecte principalement le goût. Dès les premiers essais de pasteurisation du vin en bouteilles pratiqués bien avant PASTEUR, par APPERT en 1823, repris ensuite par GERVATS en 1827-29 et par VERGNATTE et LAMOTTE en 1850, on reconnaît le besoin de n'opérer le chauffage qu'au bout de 8 jours au moins après le soutirage, lorsque le vin, étant au repos, a abandonné l'air qui s'y est dissous pendant l'opération du remplissage. Faute de 
cette précaution le vin prend un goût résiné et les bons vins sont particulièrement exposés à cette altération.

KUHN recommande même de pratiquer le chauffage des vins sous une pression supérieure à la pression atmosphérique pour permettre au vin de conserver tous les gaz qu'il renferme dans le foudre d'où on le soutire.

Or cette circonstance, ainsi que l'absence d'aération se trouvent précisément réalisées lorsqu'on pratique la pasteurisation en circulation continue de n'importe quel liquide, lait, vin ou jus de fruits, par le procédé de la couche mince et en utilisant comme moyen de propulsion la pompe à engrenage.

\title{
LA GRÉATION DE LABORATOIRES VÉTÉRINAIRES EN BULGARIE
}

\section{Leur objet. - Leur nécessité}

\author{
par K. KATRANDJEFF
}

Chef de Service à l'Institut Bactériologique Vétérinaire de Sofia

Lorsque Bourgelat fonda à Lyon la première Ecole Vétérinaire, sans doute pensait-il à créer un lien entre la médecine humaine et la médecine des animaux, celle-là devant puiser chez celle-ci des comparaisons utiles, des exemples féconds, mais il ne pouvait déjà se douter du grand rôle que la médecine vétérinaire aurait à jouer plus tard dans l'hygiène publique.

Il est indubitable que la solidité de l'armature de l'enseignement vétérinaire met aujourd'hui notre confrère à même d'intervenir d'une façon opportune, d'une part, dans la sauvegarde de l'hygiène publique, d'autre part, dans toutes les industries qui se rapportent aux productions animales. II y a d'ailleurs un lien très étroit entre l'un et l'autre de ces points, puisque l'alimentation humaine emprunte considérablement aux animaux, qu'il s'agisse de la viande, apprêtée ou non, qu'il s'agisse du lait et des dérivés de celui-ci.

Depuis la guerre, les animateurs du Service vétérinaire en Bulgarie se sont aperçus eombien il était utile, nécessaire même, de développer dans ce pays tout ce qui se rapporte aux industries animales, mais en même temps, et la question s'imposait évidemment à leur attention, ils ont senti que rien de bien ne pouvait être fait dans cette direction sans être aecompagné d'un contrôle hygiénique efficace de tous les produits qui s'y rapportent.

En Bulgarie, l'organisation des services vétérinaires est telle que l'on peut concevoir la constitution d'un cadre solide de fonctionnaires éclairés chargés du susdit contrôle.

Il ne faut point oublier que la Bulgarie est un Etat uniquement 